\title{
THE DUAL ROLE OF CHROMATIC BACKGROUNDS IN COLOR PERCEPTION
}

\author{
STEVEN K. SHEVell ${ }^{1}$ \\ Department of Psychology. The University of Michigan. Ann Arbor, Michigan 48104 . U.S.A.
}

(Receiced 12 December 1977: in rerised form 28 March 19781

\begin{abstract}
This investigation explores the color appearance changes resulting from a continuously presented adapting field. In every experiment, an incremental mixture of red and green monochromatic lights was superimposed on top of a steady red background field. On each experimental trial the intensities of the red background and the red increment were fixed: the subject adjusted the intensity of the green light so that the incremental mixture appeared a "pure" (neither slightly reddish nor greenish) yellow. In one experiment, the increment was a steadily viewed thin annulus seen on a larger background: in another experiment the increment was identical to the background in size and retinal location but was presented as a brief $(150 \mathrm{msec})$ flash: in the final experiment the increment was a briefly flashed thin annulus seen on a larger background.

For any fixed. relatively dim background level the intensities of the red and green increments were approximately in constant ratio over a nearly $2 \mathrm{log}$ unit range of test intensities. However. with more intense adapting fields the green light to red light incremental intensity ratio decreased as the test intensity was increased, with the ratio asymptoting at high test levels to an adaptation-intensity dependent value.

The empirical observations reject both von Kries' Coefficient Law and the notion that only spatial (and or temporal) transients contribute to color signals. The results are consistent with a "two-process" theory where the adapting field is assumed both to contribute directly to the chromatic signal and simultaneously to alter the amplitudes (but not shapes) of the spectral sensitivity functions associated with the three receptor-types of color vision.
\end{abstract}

\section{IXTRODLCTION}

How is the color appearance of a light affected by other chromatic stimulation presented nearby (or coincidentally) in space or time? Hundreds of research reports have explored this question under such topic headings as color constancy, asymmetric color matching. haploscopic matching. interocular hue shift. chromatic induction, simultaneous color contrast, and chromatic adaptation. the last term frequently used as a superordinate for all those previously listed (as well as some others). The present study is focused on the simplest type of stimulus arrangement, where a light is superimposed on a steady chromatic background field. Changes in the color appearance of the light caused by the background field are explored.

This relatively simple stimulus configuration is similar to retinal stimulation frequently occurring outside the laboratory. All three receptor-types of human color vision are stimulated to a certain degree with a specific area of the visual field having more quanta absorbed in each receptor-type than are absorbed in the surrounding retinal area. Surprisingly, this type of configuration has drawn much less attention than more complicated arrangements [e.g. the adapting field surrounds (but does not include) the test area. the adapting field is extinguished prior to presentation of a test flash, the adapting field and test field are seen through opposite eyes]. Part of the reason for this neglect may be a theoretical formula-

${ }^{t}$ Present address: Department of Behavioral Sciences. University of Chicago. 5848 S. University Ave.. Chicago. Illinois 60637. U.S.A. tion due to von Kries (1905) that has been in question for at least thirty-five years (Walters, 1942) but which is still assumed to hold (at least approximately) in many studies (cf. Jameson and Hurvich. 1972). The von Kries theory (frequently called the Coefficient Law) states that chromatic adaptation only serves to set the amplitude of the spectral sensitivity distribution associated with each receptor-type. The relative sensitivity to different wavelengths within a given receptor-type is assumed to be unaltered by chromatic adaptation. The assumed validity of the von Kries formulation may have led investigators to believe that the change in color appearance resulting from a chromatic background field is a well-understood phenomenon. This is false.

Twenty years ago. Hurvich and Jameson (1958) showed clear violations of the von Kries theory, and expressed the belief that changes in color appearance caused by chromatic adaptation could not be understood without considering both receptor sensitivity changes and incremental (or decremental) contributions to color signals. Their suggestion was that chromatic adaptation causes sensitivity changes. and the adapting field further results in specific visual signals that cannot be simply discarded when a test field is presented. They specifically proposed (Hurvich and Jameson, 1958: Jameson and Hurvich, 1959. 1961, 1964) that the color appearance of a light seen on an adapted patch of retina is due to the sum of the color signals from the light falling on that patch of retina (which may be affected by adaptation-caused sensitivity changes) and the color signals established by the adapting field.

Jameson and Hurvich (1972) later called this model 
the "two-process" interpretation, the first process being receptor sensitivity changes (which alone imply von Kries theoryl and the second process being an increase or decrease in color signals by a fixed amount determined solely by the adapting field. The second process will be called the "additive effect" to distinguish it from adaptation-related sensitivity changes. Changes in color signals resulting from the additive effect are affected not at all by the test stimulus whose color is being judged. The changes in color signals resulting from sensitivity changes are closely related to the test stimulus since sensitivity changes alter the receptor signals by a fixed proportion, not a fixed amount.

Recently, Walraven (1976) found empirical support for a special case of the two-process interpretation where the additive effect precisely cancels the visual signal due to the adapting field "leaving only the transient [i.e. incremental] component of the stimulus as effective signal for further processing [p. 294]." The experimental results reported here are in conflict with this special case. but do provide evidence for the presence of two adaptation processes. Specifically, this study shows that the effect of an adapting field can be explained in terms of its contribution to color signals and its simultaneous atteriuation of them by altering the gains operating on the three receptortypes spectral sensitivity functions.

\section{METHODS}

f pporutus

The experiments in this study required that a mixture of red and green lights be presented upon a large. steady red background field. The optical apparatus used is shown in Fig. 1. The apparatus, a four-channel Maxwellian view system. had as its light source (S) a $150 \mathrm{~W}$ xenon lamp (Osram XBO 150) mounted in housing $H$. The lamp was powered by a current-regulated Trygon $\mathrm{M} 7 \mathrm{C} 40-50 \mathrm{OV}$ power supply which was extremely stable $\left( \pm 0.02^{\circ}\right)$ and thus provided a very stable source of light. The lamp illuminated four Schoeffel GM 100 double monochromators $\left(M_{1}, M_{2}, M_{3}\right.$. and $\left.M_{4}\right)$ that could be adjusted in wavelength increments much smaller than $(\mathrm{nm}$. Four pairs of Wratten neutral density wedges $\left(W_{1}, W_{2}, W_{3}\right.$, and $\left.W_{4}\right)$ allowed independent intensity control for each channel. Light from monochromators $\mathbf{M}_{1}$ and $\mathrm{M}_{2}$ was combined at mixing cube $C_{1.2}$ and. similarly. light from $M_{3}$ and $M_{4}$ was combined at $C_{3.4}$. Vincent Uniblitz shutters $S_{1,2}$ and $\mathrm{S}_{3.4}$ were controlled electronically so that brief flashes of light could be presented at regular intervals. Field stops $F_{t .2}$ and $F_{3.1}$ were $35 \mathrm{~mm}$ slides located in the rear focal plane of lens $M L$. Beamsplitter cube $C_{1.2 .3 .4}$ merged the light from all four monochromators, and the Maxwellian lens $M L$ in combination with the other lenses in the apparatus imaged at the subject's pupil (P) the superimposed slit images from the four monochromators. The image at the pupil was smallet than $2 \mathrm{~mm}$ in size, thus pupil dilation was not necessary. (Wedges $W_{1.2}$ and $W_{3.4}$ were not used in this study.l

The present experiments required only three channels, so light from monochromator $M_{1}$ was not used. Monochromator $\mathrm{M}_{2}$ provided the red $(660 \mathrm{~nm})$ background field which was always presented continuously: thus shutter $S_{1.2}$ was either fixed open or closed (the latter in the dark adapted case). The red $(660 \mathrm{~nm})$ and green $(\$ 40 \mathrm{~nm})$ lights entering into the mixture were provided by $M_{3}$ and $M_{4}$. respectively. The subject could be given control of either $\mathrm{W}_{3}$ or $\mathrm{W}_{4}$ (via torque transmitting and receiving synchros) and thus could vary remotely the intensity of the red or green light. A black plastic viewing hood separated $F_{1.2}$. $F_{3.4} C_{1.2 .3 .4}$ and $M L$ from the rest of the apparatus. The viewing hood greatly reduced the amount of scattered light present at the subject's pupil. The subject's head was held stationary by a bite bar which was mounted in a threedimensional positioning device.

\section{Calibration}

Absolute intensity levels were measured for channels 2 . 3 and 4 at $580 \mathrm{~nm}$. During these measurements, all wedges were precisely set to positions near their minimum density these wedge settings will be called the "standard configuration"). A white diffusing surface was placed a known distance from the image formed by Maxwellian lens ML. and the illuminance on the surface then measured with a Mac. beth illuminometer. Using the method described by West. heimer (1966), these measurements allowed calculation of retinal illumination in photopic trolands for $580 \mathrm{~nm}$ light from each channel.

Immediately following the illuminometer measurements. a United Detector Technology PIN-1ODF photodiode filter combination was placed at the image formed by the Maxwellian lens. The PIN-10DF photodiode/filer produces a flat spectral response $\left( \pm 5_{0}^{\circ}\right)$ from 450 to greater than $900 \mathrm{~nm}$. Before altering the configuration of the apparatus. a photodiode intensity measurement was taken for the $580 \mathrm{~nm}$ light from each channel.

The experiments never required $580 \mathrm{~nm}$ light, but this wavelength was chosen in order to minimize the color difference between the fields brightness matched with the Macbeth illuminometer. With the intensity known at $580 \mathrm{~nm}$. the intensity in photopic trolands at any other wavelength $;$ is determined by the energy and photopic sensitivity at $i$ relative to the energy and photopic sensitivity at $580 \mathrm{~nm}$. For each channel, the relative energy at $i$ was measured by dividing the photodiode measurement at $i$ by the photodiode measurement at $580 \mathrm{~nm}$. Relative photopic sensitivity was determined from standard tables (Wyszecki and Stiles, 1967). With all wedges in the standard configuration. the intensities of channels 2.3 and 4 at their specified wavelengths were 2.59. 2.61 and $3.64 \mathrm{log}$ td. respectively (all logarithms are base 10 unless noted). The Wratten neutral density wedges were calibrated using the photodiode at each wavelength of light they transmitted.

The Schoeffel monochromators were the major contributors to day-to-day variability of light intensities. The apparatus was used daily by other investigators. so at the beginning of every experimental session the monochroma. tors had to be reset to the appropriate wavelengths. Uniortunately, the intensity of light from the monochromators

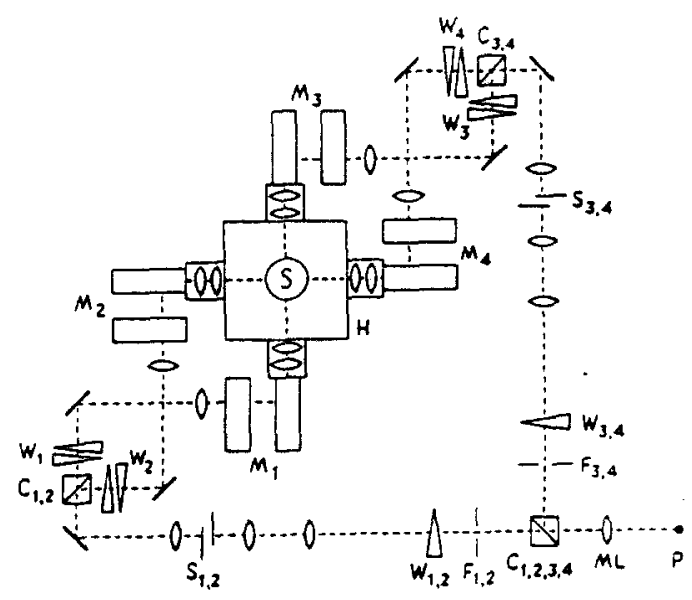

Fig. I. Schematic diagram of the apparatus. 
depended (among other things) on the direction and speed of approach to the desired wavelength setting. Much of the variability was eliminated by always approaching wavelengths from the short-wavelength end of the spectrum (this procedure was used in the intensity calibrations described above). In order to further reduce day-to-day variation. after setting the monochromators to the required wavelengths the wedges were set to the standard configuration and the daily relative intensity of each channel was measured with the photodiode. Balancing wedges at $W_{2}$. $\mathrm{W}_{3}$ and $\mathrm{W}_{4}$ then were adjusted to compensate for daily variations.

The duration of flashed stimuli was measured by connecting the photodiode to an oscilloscope (Tektronix 453) with a calibrated time-base. Only one flash duration was used (150 msec). No day-to-day variability of flash duration was detectable. The $150 \mathrm{msec}$ measurement depended on the absolute calibration of the oscilloscope which is probably on the order of $\pm 10^{\circ}{ }_{n}$

\section{Procedure}

Three experiments were conducted. The experiments varied only the spatial and temporal relations between the background field and superimposed light mixture. In each experiment there were seven conditions corresponding to seven intensities (one of which was zero) of the red. steady background $R$. The background was always a complete circular disk on which the incremental stimuli were presented (except. of course. in the condition where $R$ was of intensity zero). The incremental red light (denoted $\Delta R$ ) and incremental green light (denoted $\Delta G$ ) were mixed prior to the field stop and shutter: thus the subject saw an incremental mixture of red and green light superimposed on top of the red background. On each trial the intensities of $R$ and $\Delta R$ were fixed. The subject's task was to adjust $\Delta G$ so that the incremental mixture appeared a "pure" (neither slightly reddish nor slightly greenish) yellow. This type of psychophysical criterion has been used quite successfully by many investigators (Jameson and Hurvich. 1955: De Valois and Walraven. 1967: Gestrin and Teller. 1969: Walraven. 1973. 1976: Larimer, Krantz and Cicerone. 1974). In the present study where $\Delta G$ is added to $\Delta R$. a trained observer easily can detect when the mixture appears slightly greenish-yellow (too much $\Delta G$ ). or slightly reddish-yellow (too little $\Delta G$ ), and can find a very small intensity range where the mixture appears pure yellow (standard errors of the mean of 5 measurements were nearly always less than $0.05 \mathrm{log}$ units).

Each experimental session began with $5 \mathrm{~min}$ of dark adaptation after which the subject adapted to a given intensity of the steady red background field for $7 \mathrm{~min}$ (in the condition where the background was absent, the dark adaptation period was extended to $12 \mathrm{~min}$ ). The $\Delta G$ increment then was presented alone on $R$. This represented the first of eight $\Delta R$ intensity levels where the subject adjusted $\Delta G$ so that the mixture (seen on $R$ ) appeared yellow: the first intensity of $\Delta R$ was zero. After the subject made five $\Delta G$ intensity settings (method of adjustment) for a given level of $\Delta R$, the $\Delta R$ intensity was increased (by about 0.30 $\log$ units\} and another set of five measurements made. $A$ complete set of measurements for one condition (i.e. one $R$ intensity in one experiment) contained 40 measurements (although often the subject found the $\Delta R=0$ case to be impossible. thus leaving 35 measurements).

During a single experimental session. one. two or three conditions were run (depending on the time available). When more than one condition was run. 5 min of dark adaptation was repeated before the $7 \mathrm{~min}$ adaptation to a new background intensity. Between conditions within a session. the subject was allowed a rest period and, oc. casionally, to light adapt. Within a day, the intensity of $R$ was always increased from one condition to the next (to minimize any possible long-term adaptation effects).
Typically. a subject required five sessions lon average $2 \frac{1}{2} \mathrm{hr}$ each) to complete a single experiment. The first two sessions usually were devoted to practice (the number of practice sessions varied somewhat between subjects and experiments. depending on the variability of the subject's data). Two subjects. both males with normal color vision. participated in this study. Each subject used his right eye. One subject (MB) was completely naive as to the purpose and theoretical framework of this research. Subject SS is the author. Both subjects had become highly trained observers during 4 months of pilot experimentation.

The subjects attempted to maintain fixation in the center of the test field. In the first experiment where the annulus to be judged was continuously visible. fixation was no problem. In the other two experiments, the steady background field aided fixation except when the background intensity was zero. Then the flashed stimuli were presented in an otherwise dark field. However. both subjects found no difficulty in judging these stimuli.

In each experiment, intense steady backgrounds possibiy could raise the threshold for seeing $\Delta R$ above the $\Delta R$ intensities used in the experiment. To determine whether this was the case, the threshold for seeing $\Delta R$ on (high intensity) $R$ was measured in each experiment. The increment threshold measurement was made following the $\Delta G$ set. tings for the most intense $\Delta R$ : thus the threshold data were taken following $5 \mathrm{~min}$ of further adaptation $10 R$ alone.

\section{RESLLTS}

\section{Spatial transient experiment}

In the first experiment. the spatial transient case. the steady red background field was a 4 : circular disk on top of which was superimposed a $60^{\circ}-90^{\prime}$ incremental annulus (see Fig. 2). The annulus was a mixture of red and green lights $\Delta R$ and $\Delta G$, and was seen in steady viewing. To allow for adaptation to the steadily viewed incremental stimuli. whenever the $\Delta R$ intensity was changed the subject made a (unrecorded) $\Delta G$ setting to which he adapted for $1 \mathrm{~min}$; the recorded measurements then followed. Six levels of background intensity $R$ were used $(-1.10$. -0.39 . $+0.39,1.03,1.78$, and $2.54 \mathrm{log}$ td). In addition, the annular $\Delta R+\Delta G$ mixture was presented with $R$ absent (no background). Under dark adaptation, the

\section{SPATIAL TRANSIENT}

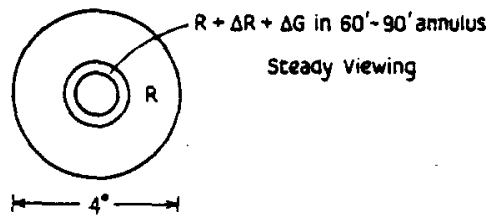

\section{TEMPORAL TRANSIENT}

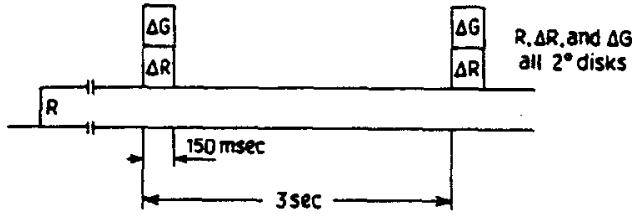

Fig. 2. Representation of the stimuli over space for the spatial transient experiment (above) and over time for the temporal transient experiment (below). 

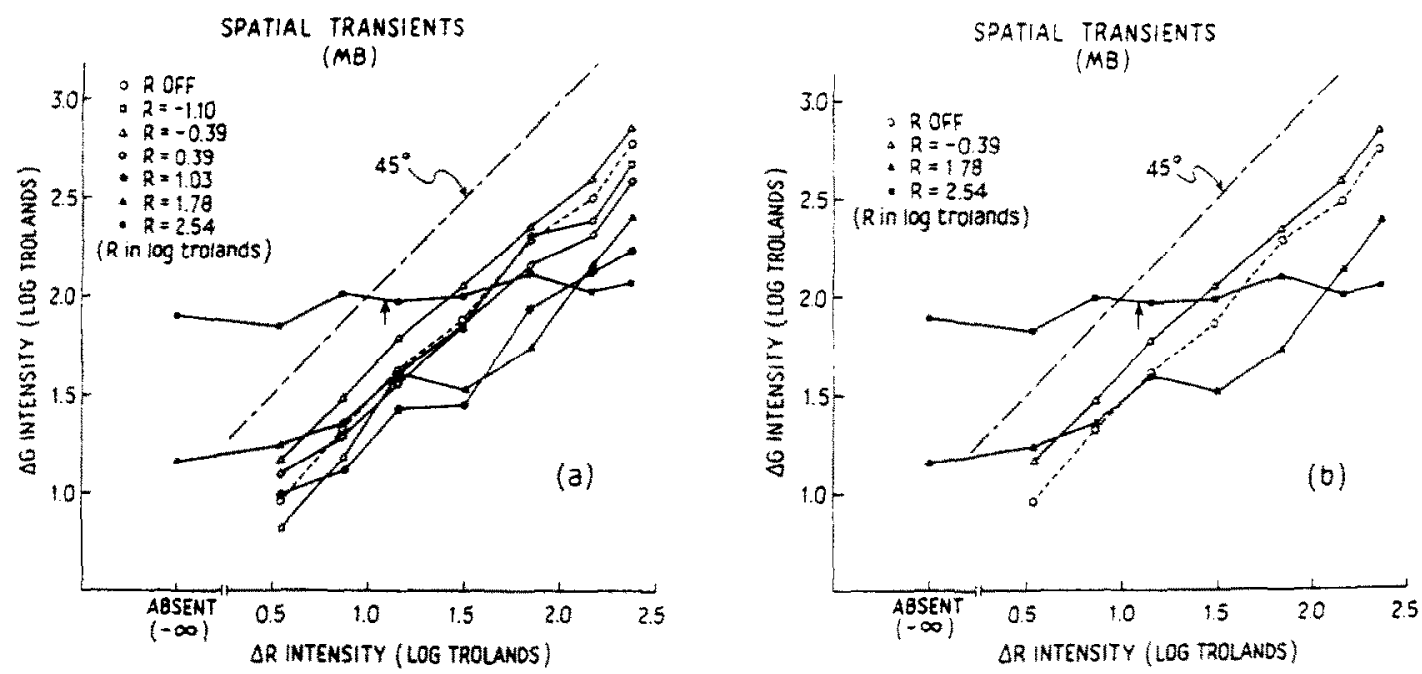

Fig. 3. (a) Incremental green light logarithmic intensity is incremental red light logarithmic intensity for the spatial transient experiment (subject $M B$ ). Each connected set of points represents a fixed red background intensity. Arrows (here and in following figures/ indicate the threshold for seeing

$\Delta R$ alone on background $R$. (b) Data for only four background conditions replotted for clarity.

Tatio of "incremental" intensities, $\Delta G \Delta R$. should be a constant since the $r-g$ chromatic response is known to be linear (Larimer. Krantz and Cicerone. 1974). This condition did not provide new empirical results but rather served as a baseline condition with which the red adaptation conditions could be compared.

The results of the experiment are shown in Fig. 3a for subject $M B$. In this plot, log $\Delta G$ intensity is plotted against $\log \Delta R$ intensity. Each data point in this figure (and all following figures) is the mean of five measurements. The dark-adapted condition is represented by the open circles connected with broken lines. A constant $\Delta G / \Delta R$ ratio in this plot would be a $45^{\circ}$ line. A reference $45^{\circ}$ line has been drawn and is closely parallel to the dark-adapted data. The data for dim backgrounds (less than 1 td) are parallel to the dark-adapted condition (open squares and triangles). As the background intensity $R$ increases. the plot of $\log \Delta G$ vs $\log \Delta R$ changes gradually from a straight $45^{\circ}$ line topen circles) to a curved line (filled triangles) and then to a nearly horizontal line (filled squares). Figure $3 b$. a replot of four conditions shown in Fig. 3a. displays the transition more clearly. Subject SS showed an identical pattern. The arrows in Fig. 3 (and in following figures) indicate the threshold intensity for seeing $\Delta R$ alone on $R$ ffor curves with no arrow. the threshold was so low that it fell off scale to the left).

One dim red background lopen triangles. Fig. 3b) shows a surprising effect. The background seems to supersensitize the eye to red light. This is indicated in the figure by the data falling parallel to but above the dark-adapted condition. In these cases. for any given $\Delta R$ intensity the red background required that

"To conserve space data for only one subject are shown for each type of plot. A complete set of figures for both subjects is given in Shevell (1977). Unless noted. the two subjects data are very similar. the dark adapted $\Delta G$ setting be increased by a fixed proportion (not a fixed amount). Thus the red background is not simply adding to the red signal. A complete discussion of this finding will be pursued in a later section. However. it should be stated here that the sensitizing effect of dim backgrounds needs to be more carefully explored to be certain that the observed findings are free of artifact te. order effects. long-term previous adaptation effectsl.

The data from the same experiment for subject SS are plotied someuhat differently in Fig. 4 in order to show more clearly the effect of increasing the background intensity $R$. $^{2}$ In Fig. 4 green light logarithmic intensity $(\log \Delta G)$ is plotted against red backyround logarithmic intensity $(\log R)$. Each set of connected points represents a fixed $\Delta R$ intensity. In the cases where $\Delta R$ was absent (and $\Delta G$ alone seen on $R$ ). the annulus would appear yellow only when $R$ was of high intensity $(\times `$. Fig. 4). For lower intensity $R$. $\Delta G$ when visible always would appear greenish.

For any fixed level of $\Delta R$ (except $\Delta R$ off), increasing $R$ does not cause a strict increase (or strict decrease) in $\Delta G$. Each set of connected points in Fig. 4 forms a curve that both rises and falls: thus increasing $R$ is neither only decreasing retinal sensitivity nor only increasing the red chromatic signal. This is (weak) suggestive support for a "two-process" theory (more exact qualitative predictions will be derived in the Discussion).

\section{Temporal transient experiment}

In the second experiment. the red steady background $R$ was reduced in size to a $2^{*}$ field. The same seven intensities of $R$ lincluding $R=0$ ) used in the spatial transient experiment were tested. The annulus of the previous experiment was replaced by a full circular disk identical to the background in size and retinal location. If the incremental mixture had been presented continuously, the light from the mixture simply would have mixed with the background to 


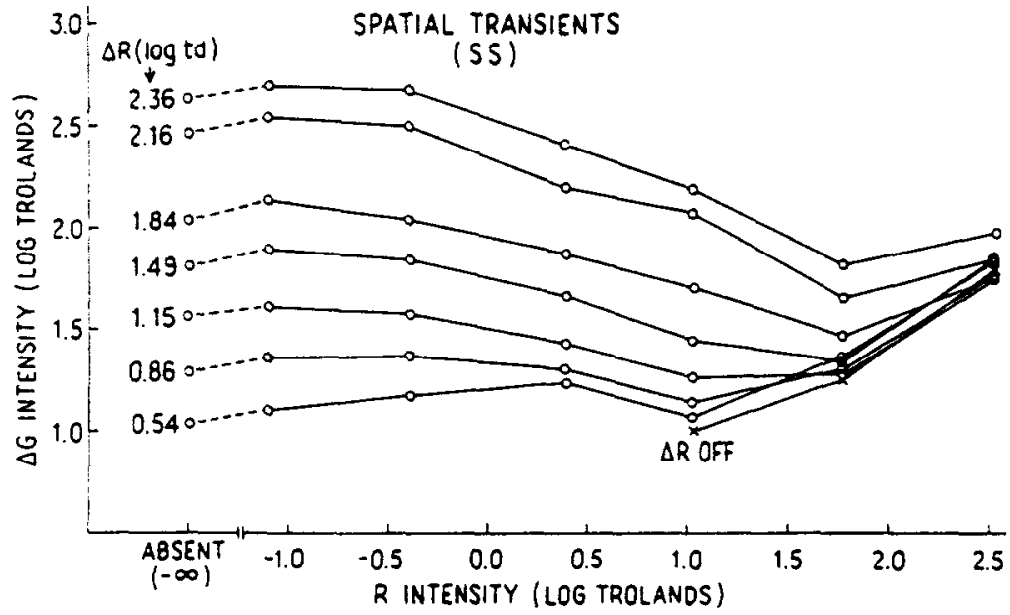

Fig. 4. Incremental green light logarithmic intensity vs red background light logarithmic intensity for the spatial transient experiment (subject SS). Each connected set of points represents a fixed intensity of the red increment $\Delta R$.

form a 2 circular field composed of $R+\Delta R+\Delta G$. However, the $\Delta R+\Delta G$ mixture was not so presented: rather the mixture was flashed for $150 \mathrm{msec}$ on top of the steady background. once every $3 \mathrm{sec}$ (see Fig. 2). As before. the $R$ and $\Delta R$ intensities were fixed and the subject adjusted the intensity of the green increment $\Delta G$ so that the $2^{*}$ field appeared a pure yellow during the $150 \mathrm{msec}$ flash. Because the incremental mixture $\Delta R+\Delta G$ was distinguishable from the background field $R$ only due to differences occurring over time. this experiment was called the "temporal transient" case.

The data from this experiment are shown in Figs 5 and 6 . For subject $M B$ (Fig. 5). $\log \Delta G$ intensity is plotted against $\log \Delta R$ intensity. The open circles connected by broken lines represent the dark-adapted condition and fall along a line of about $45^{\circ}$. Dim backgrounds give data parallel to the dark-adapted condition and. as in the spatial transient experiment. show a supersensitivity effect (the open symbols in Fig. 5 show especially clearly the supersensitivity effect for subject MB: subject SS showed little evidence for supersensitivity). As the background $R$ is increased in inlensity. the plot of $\log \Delta G$ vs $\log \Delta R$ changes from i 45 line to a curved line. Two of the curved lines (filled triangles and squares) show both a horizontal asymptote (as $\log \Delta R$ becomes small) and a 45 asymptote (as $\log \Delta R$ becomes large). In general. for both subjects, increasing $R$ tended to lower the $45^{5}$ asymptote (except for the supersensitivity effect) and either resulted in more curvature at the left end of the plot or extended the length of the horizontal asymptote.

The data for subject SS are shown in Fig. 6. plotted as $\log \Delta G$ vs $\log R$. As in the first experiment. both subjects found that an intensity of $\Delta G$ such that it alone appeared yellow on $R$ could be found only when $R$ was quite intense ( $x$. Fig. 6). All of the curves tend to converge as the background intensity becomes quite high.

\section{Spatial and temporal transient experiment}

In the third experiment. the steady red background field was 4 (as in the first experiment). The incremental mixture $\Delta R+\Delta G$ was a $60^{\prime}-90^{\prime}$ annulus flashed in the center of $R$ for $150 \mathrm{msec}$ once every $3 \mathrm{sec}$. Thus the spatial features of the first experiment were combined with the temporal arrangement of the second experiment. The red background intensities were as before, and for each given level of $R$ and $\Delta R$ the subject adjusted the intensity of $\Delta G$ so that the annulus appeared yellow during the $150 \mathrm{msec}$ flash. This experiment was called the "spatial and temporal transient" case.

The results of this experiment are displayed in Figs 7 and 8. As before. the dark-adapted condition (broken line) in Fig. 7 follows approximately a $45^{\circ}$ line. The curves begin to deviate from a straight $45^{\circ}$ line at background intensities higher than in the pre. vious experiments. For subject MB (Fig. 7), the highest intensity background $(2.54 \log$ td) does not yield

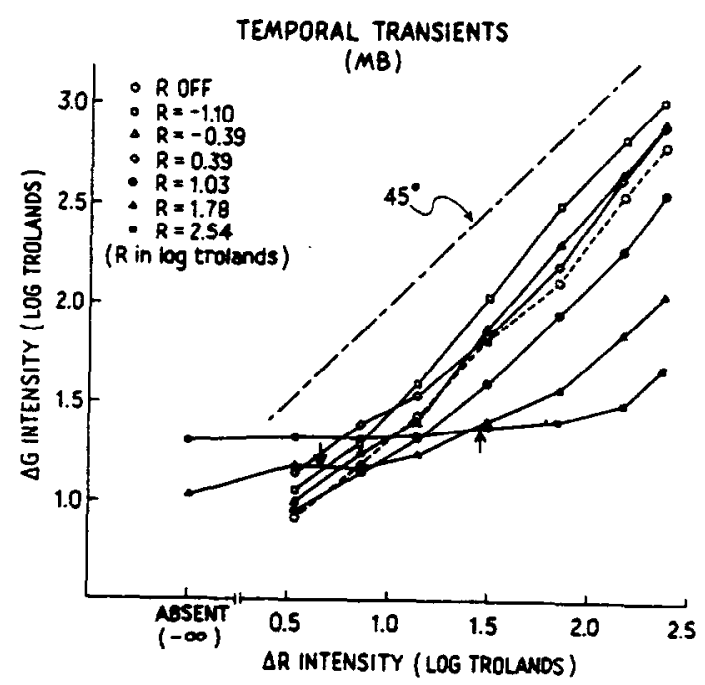

Fig. 5. Incremental green light logarithmic intensity vs incremental red light logarithmic intensity for the temporal transient experiment (subject MB). Each connected set of points represents a fixed red background intensity. 


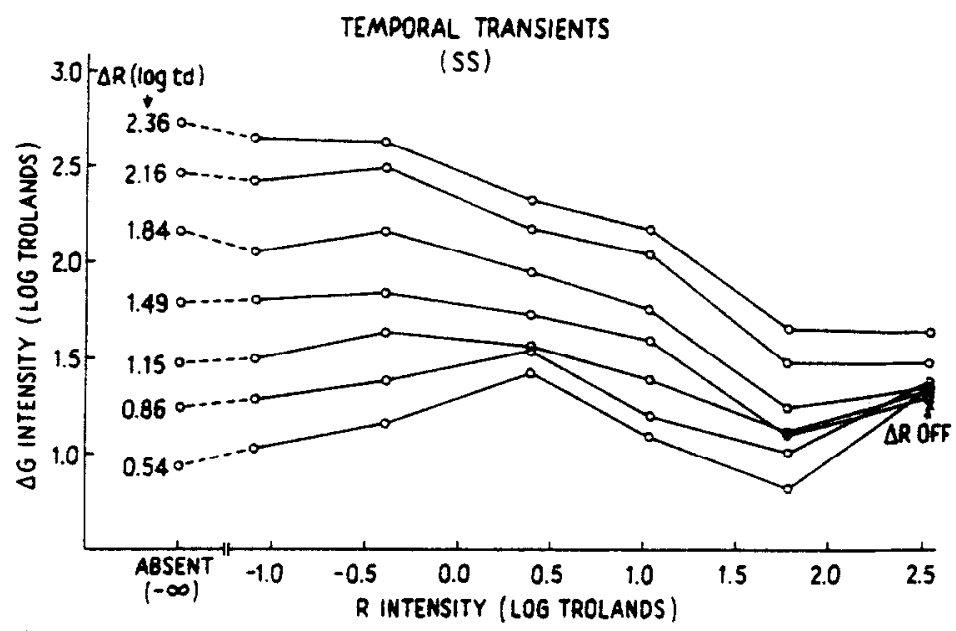

Fig. 6. Incremental green light logarithmic intensity vs red background light logarithmic intensity for the temporal transient experiment (subject SS). Each connected set of points represents a fixed intensity of the red increment $\Delta R$.

the horizontal asymptote at low $\Delta R$ levels seen in this subject's data for spatial transients or temporal transients alone (see Figs 3 and 5). Subject MB continues to show a clear supersensitivity effect: however. subject SS showed no sign of supersensitivity. While somewhat different from the previous experiments. the data for both subjects continue to show that increasing $R$ intensity tends to lower the $45^{\circ}$ asymptote seen in the curves (except when supersensitivity is observed) and, at higher levels. results in more curvature in the plots. It should be noted that at the highest background level (2.54 log td) both subjects show a surprising result. Low intensities of $\Delta R$ still below threshold (see arrow on curve connecting filled squares) appear to increase the intensity of $\Delta G$ required to make the flashed annulus appear yellow.

Figure 8 shows the data for subject SS plotted as $\log \Delta G$ against $\log R$. While the curves are tending to converge as $R$ goes to its highest intensity. the curves are much more dispersed than in the previous two experiments (compare Figs 4 and 6).

\section{DISCLSSION}

The most general empirical result from these data can be described by examining the ratio of incremental intensities. $\Delta G \Delta R$, as $\Delta R$ is varied and the background intensity $R$ is held fixed. When the incremental mixture is presented on a given, relatively dim background, $\Delta G_{i} \Delta R$ is approximately constant: however. when the background level is more intense the ratio declines as $\Delta R$ is increased. This general result is found (for both subjects) whether $\Delta R$ and $\Delta G$ are increments over space or time or both (although to a lesser degree in the last case). It is not dependent on the test field being surrounded by a much larger area of adapted retina. The result is indicated in Figs 3. 5 and 7 (for subject $M B$ ) by the plots that change

\footnotetext{
${ }^{3}$ Data from subject $M B$ also show clear deviations from a $45^{\circ}$ line. though they do not show the same pattern as subject SS.
}

from a straight $45^{-}$line to curved lines as background intensity $R$ becomes larger.

\section{Comparisons with theoretical formulations}

The present data confirm previous evidence (MacAdam. 1956: cf. Jameson and Hurvich. 1972) indicating the inadequacy of von Kries Coefficient Law. The Coefficient Law requires that all light in the test area (whether due to the background or superimposed test field) be summed. implying

$$
\log \Delta G=\log (\Delta R+R)+\log g(R) .
$$

Thus for any fixed intensity of background $R$. a plot of $\log \Delta G$ vs $\log (\Delta R+R)$ should follow a $45^{\circ}$ linc. Figure 9 shows that the data from the spatial transient experiment (subject $\mathrm{SS}^{3}$ ) systematically deviate from the dashed reference lines drawn at 45 .

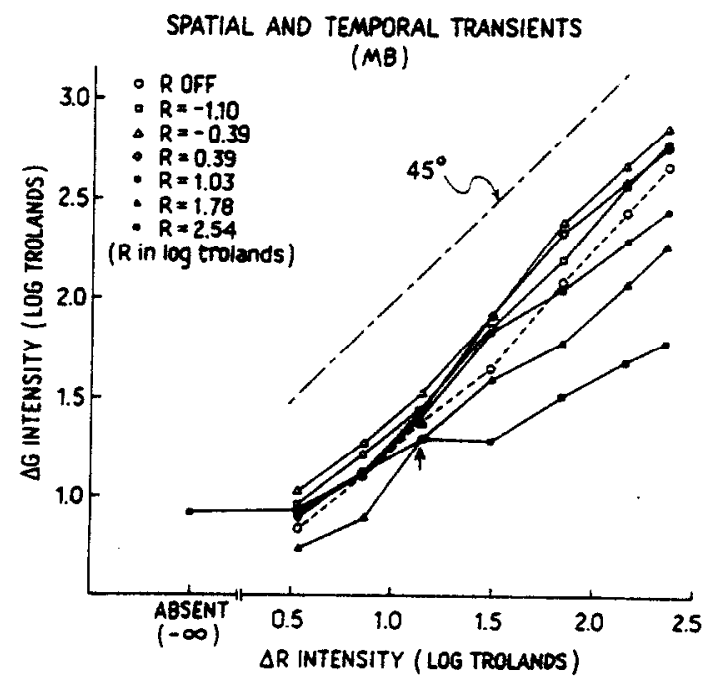

Fig. 7. Incremental green light logarithmic intensity vs incremental red light logarithmic intensity for the spatial and temporal transient experiment (subject MB). Each connected set of points represents a fixed red background intensity. 


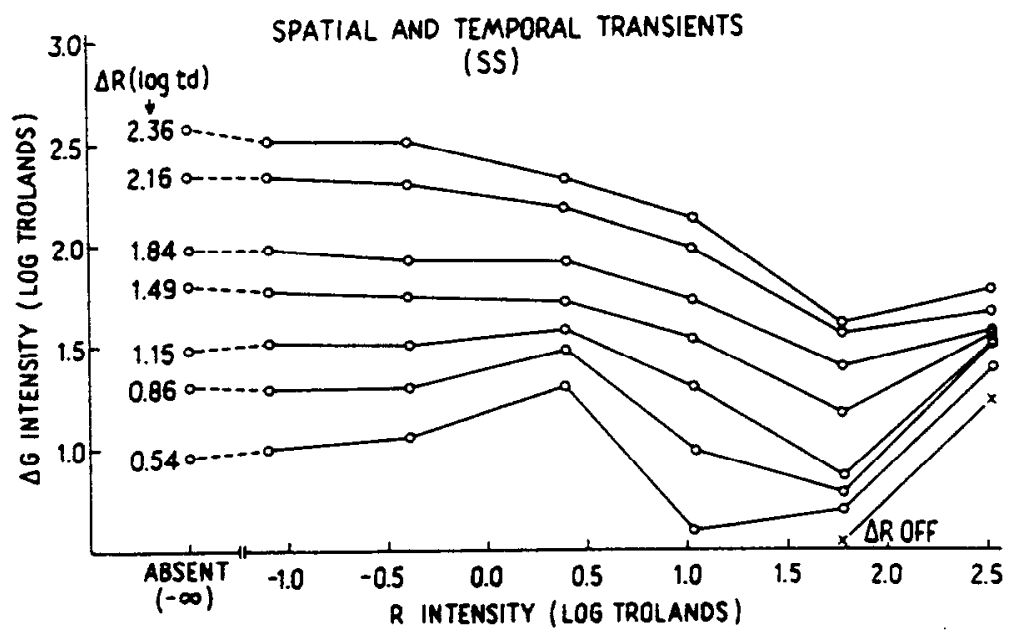

Fig. 8. Incremental green light logarithmic intensity vs red background light logarithmic intensity for the spatial and temporal transient experiment (subject SS). Each connected set of points represents a fixed intensity of the red increment $\Delta R$.

The results of this study also are in conflict with the proposal offered by Walraven (1976) that states the background field $R$ results only in a color-selective sensitivity change and that the quanta from the background light falling in the test area have no further effect on the perceived color. That is, the sensitivity change is applied only to the increments $\Delta R$ and $\Delta G$; quantitatively this implies

$$
\Delta G=\Delta R g(R) \text {. }
$$

Empirical support for this formulation was found in an experiment very similar to the spatial transient experiment described here. Walraven interpreted his results to indicate "that only the incremental stimulus components contribute to color perception [p. 291]." Whittle and Challands (1969) have suggested a similar theory for bright ness contrast that emphasizes the importance of spatial and temporal transients.

The present study clearly demonstrates that for color contrast this view is incorrect. The most direct demonstration that backgrounds do contribute to color perception is the fact that an incremental, steadily viewed green annulus $\Delta G$ seen on an intense red background $R$ can be adjusted so that $\Delta G$ (alone) seen on $R$ appears yellow ( $\times$ 's, Fig. 4). Walraven's

t The qualitative argument against Walraven's formulation was formalized by computing a two-way $(7 \times 7)$ analysis of variance $(7$ background intensities $\times 7$ non-zero $\Delta R$ intensities: the analysis of variance assesses Eqn 2 on the assumption that $\Delta R=0$ is an extraordinary limiting case, since the data collected with $\Delta R$ off alone are sufficient to reject Eqn 21 with repeated measures (this is the only analysis in which the data for both subjects were analyzed together). According to Eqn 2 .

$$
\log \Delta G=\log \Delta R+\log g(R)
$$

in which case there should be no $\Delta R \times R$ interaction. Comparing the mean square for interaction to the appropriate error term yielded an $F$-ratio that implies the interaction between $\Delta R$ and $R$ is significantly different from zero $\left(F_{36.36}=22.3, p<0.001\right)$. Thus Eqn 3 is rejected. theory specifies that no intensities of $\Delta G$ and $R$ can appear yellow when $\Delta R$ is absent. The curved and horizontal lines in plots of $\log \Delta G$ vs $\log \Delta R$ (Figs 3,5 and 7) further demonstrate that the red chromatic background cannot be assumed not to contribute to the color signal. ${ }^{4}$

The discrepancy between the present experimental results and those of Walraven (1976) may be explained in part by a difference in procedure. Walraven fixed $\Delta G$ and had the subject adjust $\Delta R$, while here $\Delta R$ was fixed and the subject adjusted $\Delta G$. Walraven found that at high $R$ intensities. measurements with much lower (fixed) $\Delta G$ intensities were imposs. ible because the annular area always appeared red.

\section{SPATIAL TRANSIENTS (SS)}

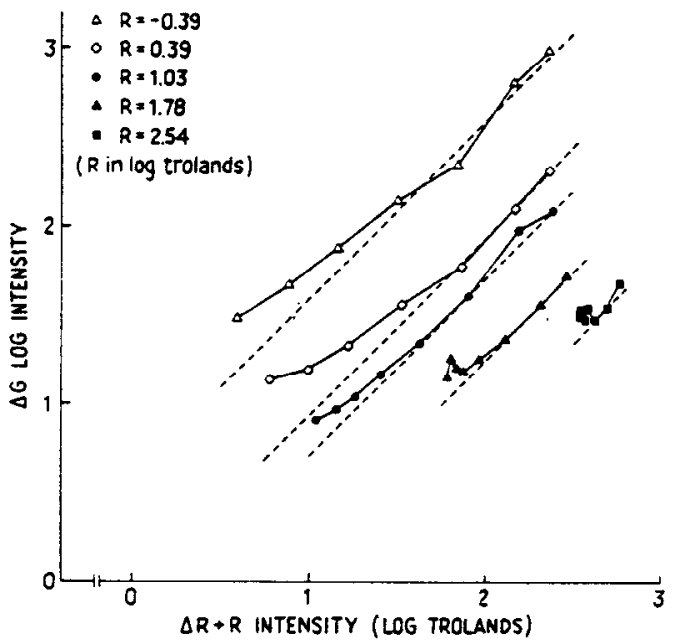

Fig. 9. Incremental green light logarithmic intensity vs total red light $(\Delta R+R)$ logarithmic intensity for the spatial transient experiment (subject SS). Each connected set of points represents a fixed red background intensity. The dashed lines have been drawn at $45^{\circ}$ for reference (the curves have been shifted vertically an arbitrary amount for clarity). 


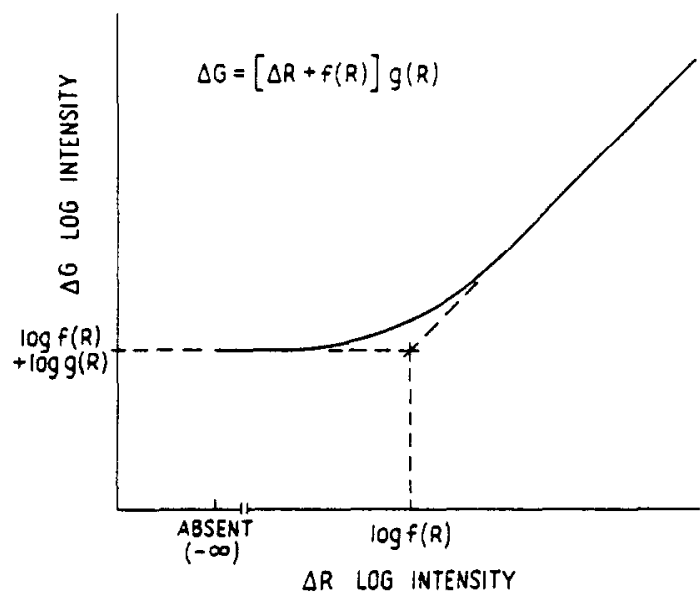

Fig. 10. A plot of $\log \Delta G$ vs $\log \Delta R$ according to the theoretical equation $\Delta G=[\Delta R+j(R)] g(R)$.

Since in his experiment the subject controlled $\Delta R$ and not $\Delta G$. the subject could not add more green light to cancel the redness. At higher background intensities. Walraven simply stopped collecting data "at a certain minimum value of $\Delta G$, because at the next lower value of $\Delta G$ the test field [was] below (increment) threshold [p. 291]." This is why the curves in his Fig. 3 for the four highest $R$ intensities have fewer data points than the others. Since Walraven used $\Delta G$ intensity steps of about $0.5 \mathrm{log}$ units, he simply may have skipped over the horizontal and curved portions of the curves. His truncated curves for 2.43 and 2.77 log td background fields possibly show the beginning of deviation from a $45^{\circ}$ line, but in this regard the data are too noisy and too few to be sure (note that in his Fig. $3 \log \Delta R$ is on the ordinate and $\log \Delta G$ on the abscissa, so data comparable to those found in the present study would approach a vertical rather than horizontal asymptote).

What remain unresolved are differences between the two studies for background intensities in the range 1.0-2.8 log td. Comparing Fig. 3 of this study with Walraven's Fig. 3. the present study shows clear deviations from a 45 line when Walraven's data do not. The excellent agreement in this regard seen here between observers and between experiments leaves no doubt that the data reported here are replicable. Similar results from a large number of pilot experiments further serve to verify the repeatability of these measurements.

The presence of an additive effect. as demonstrated by the fact that mixtures of $\Delta G$ and $R$ appear yellow. suggests a generalization of Walraven's and von Kries' theories, where

$$
\Delta G=[\Delta R+f(R)] g(R)
$$

\footnotetext{
${ }^{5}$ For any given set of experimental conditions and background intensity when $f(R)$ and $g(R)$ are constants. Eqn 4 is formally identical to the Weber-Fechner function $\Delta I=k\left(I+I_{0}\right)$.

${ }^{6} \mathrm{~A}$ complete description of the parameter estimation procedure and the actual parameter estimates are given in Shevell (1977).
}

or, equivalently,

$$
\log \Delta G=\log [\Delta R+f(R)]+\log y(R) .
$$

here $f(R)$ and $g(R)$ are functions of only the background intensity $R$. Thus, the parameters $f(R)$ and $g(R)$ represent an additive effect and sensitivity changes. respectively. resulting from the background. The curve implied by Eqn 4. plotted in log-log coordinates, is shown in Fig. 10. It easily can be shown (Shevell. 1977) that when $\Delta R \ll f(R)$ the curve approaches a horizontal asymptote, and when $\Delta R \gg f(R)$ the curve approaches a $45^{\circ}$ asymptote. Further. the two asymptotes intersect at the point $[\log f(R) . \log f(R)+\log g(R)]$ (see Fig. 10 ).

It also can be demonstrated that changes in $f(R)$ and $g(R)$ only alter the horizontal and vertical position of the curve plotted in Fig. 10. The shape and orientation of the curve are invariant. Thus the parameters $f(R)$ and $g(R)$ only serve to slide the curve parallel to the coordinate axes. Note that the $f(R)$ and $g(R)$ values may translate the curve (horizontally and vertically) so that the data follow only the asymptotic horizontal or 45 portion of the curve (the curved portion would be translated beyond the fixed range of $\Delta R$ values used in an experiment). For example. When $R$ is dim one would expect $f(R)$ to be small and thus $\log f(R)$ may be a large negative number: in that case the curve of Fig. 10 would "slide down" the $45^{\circ}$ asymptote tending to move the horizontal and curved portions of the plot below the lowest $\Delta R$ intensity used in an experiment."

The template curve of Fig. 10 was fit to each of 42 sets of data (2 subjects $\times 3$ experiments $\times 7$ background intensities) by a nonlinear optimization routine. The computer program, called PRAXIS (Brent, 1973). searched a two-dimensional parameter space seeking the values of $\log f(R)$ and $\log g(R)$ that minimized the sum of squared deviations between observed and predicted values of $\log \Delta G$.

The parameter estimates ${ }^{6}$ were generally consistent with what would be expected. For subject MB. increasing $R$ always resulted in a larger additive effect $f(R)$. Subject SS showed the same general pattern, although there were a few exceptions. For $R$ increasing beyond $-0.39 \mathrm{log} t d$. greater $R$ intensity reduced $g(R)$ in every case, indicating sensitivity to red light was declining as $R$ became more intense. For both subjects, the parameter estimates indicated that a given high intensity of $R$ provided a smaller additive effect $f(R)$ in the spatial and temporal transient experiment than in the others. This is consistent with the greater dispersion of points when $R=2.54 \mathrm{log}$ td seen in Fig. 8 as compared to Figs 4 and 6 .

For each condition. the fit of the data to the theoretical predictions can be displayed graphically by plotting together the curve of Fig. 10, adjusted by the computer-determined parameters $\log f(R)$ and $\log$ $g(R)$, and the empirical observations. Such comparisons are plotted in Fig. 11 for subject $\mathrm{MB}$. The figure shows that the theoretical structure expressed by Eqn 4 characterizes quite accurately the data from the various conditions of each experiment. For subject $\mathrm{MB}$, the square root of the average lover all three experimentsl of the squared deviation was $0.067 \mathrm{log}$ td. For subject SS, this measure was $0.061 \mathrm{log}$ td.

Figure 11 shows that, for any fixed background 
level. the quantitative relationship between $\Delta G$ and $\Delta R$ can be expressed accurately by Eqn 4 . A further assessment of Eqn 4 may be made by comparing $\Delta G$ measurements for a single (fixed) $\Delta R$ level across different intensities of the background field (i.e. across. rather than within. levels of $R$ ). Viewing the data in this way allows evaluation of whether the additive effect has a sensible quantitative relationship with background intensity (in Fig. 11, $f(R)$ may be any value for any level of $R$. so long as a good fit is obtained). Equation 4 implies that when $\Delta R$ is small (and fixed), increasing $R$ should make the $\Delta R$ value less significant on the assumption that $f(R)$ is increasing with $R$. Thus one might expect $\Delta R=0$ and relatively low $\Delta R$ intensity curves to be coincident when $R$ is large. This is exactly what is observed in Figs 4 and 6 , comparing the $\times$ 's and the curve for $\Delta R=0.54 \log t \mathrm{~d}$. However, $R=0.54 \mathrm{log} t \mathrm{~d}$ is subthreshold for the highest intensity background (see arrows, Figs 3 and 5 ). What is more important is the convergence of data points for all $\Delta R$ intensities at the most intense background level. For larger $\Delta R$ intensities it may not be assumed that $\Delta R$ is insignificant relative to $f(R)$. so one would expect the higher intensity $\Delta R$ curves to be somewhat above the rightmost " $x$ " in Figs 4 and 6 . What is clear, though. is that the red increment (even at $2.36 \mathrm{log}$ td) raises the required $\Delta G$ intensity very little when the increments are seen on an intense background.'

Another qualitative implication of Eqn 4 is that when $\Delta R$ is fixed and large relative to $f(R)$ then the effect of changing background intensity $R$ mostly will be due to changes in $g(R)$. Consider two large, fixed $\Delta R$ intensities $\Delta R_{1}$ and $\Delta R_{2}$. According to Eqn 4. the respective levels of $\Delta G\left(\Delta G_{1}\right.$ and $\left.\Delta G_{2}\right)$ should be in the ratio

$$
\frac{\Delta G_{1}}{\Delta G_{2}}=\frac{\left[\Delta R_{1}+f(R)\right] g(R)}{\left[\Delta R_{2}+f(R)\right] g(R)} \approx \frac{\Delta R_{1}}{\Delta R_{2}}
$$

assuming $\Delta R_{1} \gg f(R)$ and $\Delta R_{2} \gg f(R)$. Equation 5 specifies that curves in Figs 4.6 and 8 for high $\Delta R$ intensities should be parallel and separated by their difference in $\Delta R$ logarithmic intensities, at least until the intensity of $R$ increases to the point where $\Delta R \gg f(R)$ is invalid. This pattern is clearly evident for the three curves in each of Figs 4,6 and 8 where $\Delta R$ is $1.84 \mathrm{log}$ td or larger. As expected, the parallelism begins to deteriorate as $R$ becomes large and $f(R)$ is no longer insignificant relative to $\Delta R$.

Equation 4 would provide a most parsimonious formulation if the quantities $f(R)$ and $g(R)$ depended only on the background intensity $R$ and were completely independent of the temporal and spatial relationships between the background field and the incremental light seen upon it. The data. however. do not support this proposition. A likelihood ratio statistical test of hierarchical models explored whether either $f(R)$ or $g(R)$ could be assumed not to depend

The spatial and temporal transient experiment (Fig. 8) shows less (though some) convergence of points at $R=2.54 \mathrm{log}$ td. This suggests that $f(R)$ is not increasing with $R$ as rapidly as in the other experiments. a conjecture that was supported by the computer-determined estimates of $f(R)$.
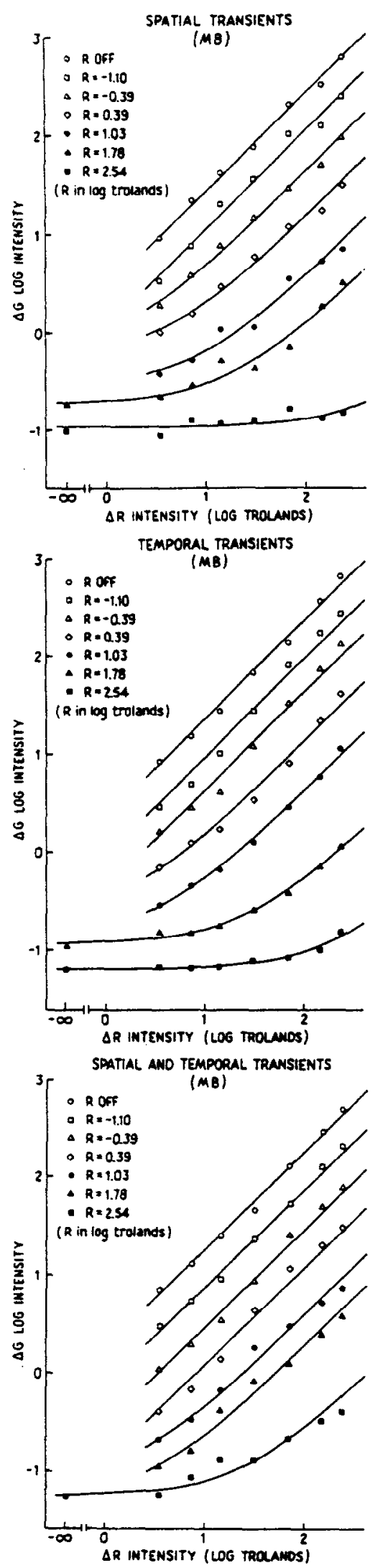

Fig. 11. Comparisons between the empirical observations of Figs 3.5 and 7 and the theoretical predictions. The curves have been shifted vertically an arbitrary amount to improve clarity. 
on whether the increment was a spatial transient. a temporal transient, or both. For each subject. the data implied extremely large $\%^{2}$ values that leave little doubt $(p<0.001)$ that models where either $f(R)$ or $g(R)$ does not depend on transient-type must be rejected (for details see Shevell. 1977).

In summary. then, the data give strong support to the model specified by Eqn 4 where a background field both contributes to the chromatic signal and attenuates it. The experimental results further show that $f(R)$ and $g(R)$ depend on the experimental conditions as well as the background intensity. The fact that a given adapting field has an effect that depends on the parameters of the incremental stimulus surely is not a new result. This is precisely the fact that led Crawford (1947) to express dark-adaptation threshold measurements in terms of an equivalent background intensity.

\section{Supersensiticit!}

The data presented in Figs 3.5 and 7 showed that a dim red background field appears to increase rather than decrease sensitivity to red light. The effect was most prominent for subject MB who demonstrated supersensitivity in all three experiments: the data for subject SS indicated clear supersensitivity only in the spatial transient experiment. While a red background might contribute redness to the mixture, such a contribution should become less important as the $\Delta R$ intensity is increased. The parallelism between the dark-adapted data and the dim background data shows that the low intensity backgrounds were affecting the required $\Delta G$ settings by a fixed proportion, not a fixed amount. Compared to the dark-adapted case, for subject MB (at least) dim red background fields increased relative red light to green light sensitivity.

Although the supersensitivity finding appears to be quite reliable across experiments for subject $M B$. the present study was not designed to explore this. and thus the data indicating supersensitivity were not collected in such a manner so as to exclude possible experimental artifact. The most obvious possible artifact is an order effect: to eliminate possible long-term adaptation effects, the dark-adapted condition always was run at the beginning of an experimental session. Another possible criticism is the absence of a fixation point when no background was present and the incremental mixture was flashed (this is not relevant to the spatial transient experiment and, further. seems an unlikely problem because spatial uncertainty concerning the location of the flashed mixture would seem to have little effect on the color perception task used here). A third possibility is related to the order effect. In the dark-adapted condition, the green $\Delta G$ "increment" was presented at intensities up to about $3 \log$ td. If the green light resulted in very long-term adaptation effects and such unexpected effects were not compensated by adaptation due to $\Delta R$, then sensitivity to red light could be relatively enhanced in subsequent conditions. This possibility also is unlikely since (1) the dim background conditions were frequently (but not always) the first condition run on a given day, and (2) a $12 \mathrm{~min}$ period (minimum) of dark and red light adaptation separated conditions run within a session.
It should be emphasized that the supersensitivity finding seen here is quite small relative to the adaptation effects resulting from more intense background fields.

\section{The two-process theories}

Since the empirical results from both subjects in each of the three experiments are accurately described by Eqn 4, it is valuable to consider the general theoretical viewpoints that imply Eqn 4 . Equation 4 is a direct implication of the Jameson and Hurvich (1972) "two-process" interpretation which is based on Hering's (1878) opponent-colors theory. Attention here will be restricted to the red-green chromatic response which, under dark adaptation. has been shown to be a linear combination of the receptor-types spectral sensitivities: thus in the dark, the red-green $(r-g)$ response is

$$
\mathrm{I}-\mathrm{g}=\sum_{i}\left[e_{i}\left(a_{1} x_{i}-a_{2} \beta_{i}+a_{3} \gamma_{i}\right)\right]
$$

where the $a_{i}$ 's are weighting constants for the cones spectral sensitivities $\alpha_{;}, \beta_{;}$and $; ;$. To be clear. Eqn 6 is an empirically verified relationship between the red-green opponent and the receptor-types spectral sensitivities: it is not a hypothesis (Larimer et al.. 1974).

The first process of chromatic adaptation is expressed by the $a_{i}$ coefficients in Eqn 6 . An adapting light is assumed to differentially alter the amplitudes of the three spectral sensitivity curves. The second process in the "two-process" interpretation is the additive effect which alters chromatic signals by a fixed amotint. The additive effect is absent in Eqn 6 because it is zero under dark adaptation (i.e. $f(0)=0)$.

A number of different theoretical assumptions concerning the level of signal processing at which the additive effect enters all imply Eqn 4 . These formulations will be referred to by the general term "two-process theories." The Jameson and Hurvich (1972) model specifies that under chromatic adaptation. the additive effect enters after the linear chromatic response $(\mathrm{r}-\mathrm{g})$ has undergone a nonlinear transformation. That is.

$$
\begin{aligned}
(\mathrm{r}-\mathrm{g})^{*}= & f^{*}\left[\sum_{i}\left(e_{i}\left(a_{1}(R) x_{j}-a_{2}(R) \beta_{i}+a_{3}(R) y_{i}^{\prime}\right)\right]\right. \\
& +K_{t-\mathrm{g}}(R)
\end{aligned}
$$

where each $a_{i}(R)$ explicitly indicates its dependency on $R, f^{*}$ is an unspecified nonlinear function. $K_{r-x}(R)$ is the additive effect of the adapting stimulation, and $(\mathrm{I}-\mathrm{g})^{*}$ is perceiced redness $/ g r e e n n e s s$. The function $f^{*}$ is a transducer function that converts the (linearly summed) chromatic response to a perceived quality of the stimulus. An analogy is the cube root function frequently applied to the (linearly summed) luminance of a stimulus: the cube root function is assumed to relate luminance to perceived brightness just as $f^{*}$ is assumed to relate the $\mathrm{r}-\mathrm{g}$ chromatic response to perceived redness/greenness.

It can be shown (Shevell, 1977) that this formulation (Eqn 7) leads to Eqn 4, provided the transducer function $f^{*}$ is monotonic and continuous. However. a transducer function as simple as $f^{*}$ now appears 
incapable of expressing the relationship between the linear chromatic response and the magnitude of perceived response (Krantz 1975: Moeller. 1976). It may well be the case that perceived redness greenness depends, for example, on "veiling" effects (that result from the yellow blue chromatic response and whiteness) as well as the $r-g$ chromatic response.

Because of the empirical evidence in conflict with Eqn 7. the alternative model

$\mathrm{r}-\mathrm{g}=\sum_{i}\left[e_{i} i a_{1}(R) \alpha_{i}-a_{2}(R) \beta_{i}+a_{3}(R) \eta_{i j} j\right]+K_{\mathrm{r}-4}(R)$

is suggested. Here the additive effect enters directly into the chromatic response of Eqn 6. Equation 8 provides a simple theory that is a minimal generalization of the proved relationship (Larimer et al. 1974) between $r-g$ and the receptor-types' spectral sensitivities (Eqn 6). defining $K_{r-1}(0)=0$. Since the wavelength of the green light is sufficiently long to assume that the short-wavelength sensitive cones contribute insignificantly to the appearance of the mixture, the condition for an experimental setting that appears neither reddish nor greenish is expressed by

$$
\sum_{i}\left[e_{i}-a_{2}(R) \beta_{i}+a_{3}(R) ; i\right]+K_{i-q}(R)=0 \text {. }
$$

Substituting the wavelengths of the incremental red and green lights contributing to the judged patch. and including in $e_{;}$an adjustment for ocular media absorption. Eqn 9 may be rearranged to yield

$$
\Delta G=[\Delta R+f(R)] g(R)
$$

where

$$
f(R)=\frac{K_{r-8}(R)}{\tau_{660}\left[-a_{2}(R) \beta_{660}+a_{3}(R) r_{660}\right]}
$$

and

$$
g(R)=\frac{-\tau_{660}\left[-a_{2}(R) \beta_{660}+a_{3}(R) \psi_{660}\right]}{\tau_{5+0}\left[-a_{2}(R) \beta_{540}+a_{3}(R) \psi_{540}\right]} .
$$

The terms $\tau$; represent the ocular media transmission at wavelength $i$. and $\Delta R$ and $\Delta G$ are, respectively. the intensities of the red and green lights mixed together and adjusted to appear yellow under the chromatic adaptation provided by $R$. Under this model the parameter $g(R)$ may be interpreted as the sensitivity of the $\mathrm{r}-\mathrm{g}$ chromatic response system to red light relative to the sensitivity to green light.

Another class of theoretical models can be constructed where perceived redness greenness $(\mathrm{r}-\mathrm{g})^{*}$ depends both on $r-g$ and veiling. and where the additive effect enters after a transducer function (such as $f^{*}$ in Eqn 7). Letting $\phi$ be the linearly summed $\mathrm{r}-\mathrm{g}$ chromatic response of Eqn 6 and $\xi_{1}$ and $\bar{\zeta}_{2}$ be the (possibly nonlinear) yellow blue and white/black responses. respectively, suppose the effect of veiling is expressed by letting $(\mathrm{r}-\mathrm{g})^{*}$ be a function of the two quantities

and

$$
V_{1}=h_{1}\left(\phi+\check{\zeta}_{1}\right)-h_{1}\left(\check{\zeta}_{1}\right)
$$

$$
V_{2}=h_{2}\left(\phi+\check{\zeta}_{2}\right)-h_{2}\left(\breve{\zeta}_{2}\right)
$$

where $h_{1}$ and $h_{2}$ are nonlinear compressive functions; then

$$
(\mathrm{r}-\mathrm{g})^{*}=F\left(V_{1}, V_{2}\right)+K_{\mathrm{t}-\mathrm{g}}(R) \text {. }
$$

If the additive effect $K_{\text {r-y }}(R)$ is relatively small, then it can be shown (see Appendix in Shevell. 1977) that Eqn 12 implies Eqn 4 to a close approximation when

$$
\frac{\mathrm{d} h_{1}(x)}{\mathrm{d} x} \approx c_{1} \quad \text { and } \quad \frac{\mathrm{d} h_{2}\left(y^{\prime}\right)}{\mathrm{d} y} \approx c_{2}
$$

$\left(c_{1}\right.$ and $c_{2}$ constants).

Comparison with watelength measurements of redigreen equilibrium colors

A study by Cicerone. Krantz and Larimer (1975) reports the effect of moderate $(40 \mathrm{td})$ red chromatic adaptation on the wavelengths of red/green equilibrium lights (i.e. yellow and blue lights that are neither reddish nor greenish). In their experiments a 2.6 . $650 \mathrm{~nm}$ adapting light was presented continuously except that it was extinguished during the $1 \mathrm{sec}$ period when the test flash was presented. The wavelengths of equilibrium blue and equilibrium yellow were measured for test lights of four different intensities (the range covering approximately $1.7-3.2 \mathrm{log} t d$ ). all presented on a patch of retina subjected to the identical red chromatic adapting stimulation. The data for four observers. taken from their Table 1 (p. 1127). are plotted in Fig. 12. The left side of the figure (wavelengths less than $500 \mathrm{~nm}$ ) shows equilibrium blue measurements, and the right side shows equilibrium yellow settings. The data points represent wavelength settings under the above described red adaptation: the dashed lines are each subject's (intensity invariant) dark-adapted measurements.

These data. from a paradigm quite different from that used in the present study, are independent evidence for the additive and gain change effects expressed in Eqn 4. on the assumption that a negative green afterimage (resulting from the red adapting field that is extinguished during the test field presentation) adds to the chromatic signal. Then. according to the two-process theories, the negative afterimage results in an additive effect expressed in Eqn 4 by a negative $f(R)$ value. The additive effect, of course, is accompanied by receptor sensitivity changes.

In the wavelength measurement experiments, the additive effect and the receptor sensitivity changes would be expected to act in harmony with respect to equilibrium yellow settings. Both effects tend to require more redness in the stimulus to compensate (1) for the reduced sensitivity of the long-wavelength cones. and (2) for the greenness from the afterimage. The additive effect should have its greatest influence at the lowest test intensity, thus the lowest intensity test field should show the longest wavelength equilibrium yellow. As intensity is increased the additive effect becomes less important and the measurements should move toward shorter wavelengths, finally approaching some wavelength longer than the darkadapted unique yellow setting: this wavelength represents only the receptor sensitivity changes. All four subjects show this general pattern in Fig. 12.

Further, equilibrium blue measurements made under red adaptation should show the same additive and receptor sensitivity change effects. With blue light, however, the short-wavelength cones must be considered (short-wavelength cones could be ignored in the equilibrium yellow experiments since the "blue" 


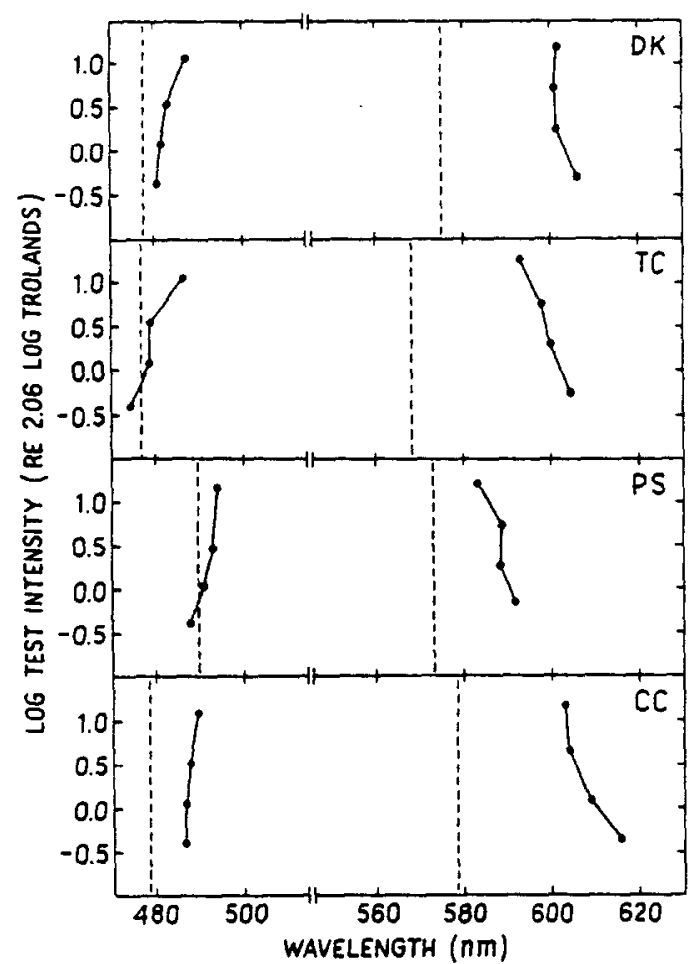

Fig. 12. Warelength measurements of equilibrium biue (left) and equilibrium yellow (right) for four observers. Data points are measurements made under moderate 140 (d) red chromatic adaptation (see text): dashed lines are dark-adapted settings. All data plotted are from Cicerone et al. (1975. p. 1127). The second lowest intensity equilibrium blue data point for subject PS corrects a typographical error in the published data.

cones are relatively insensitive to the wavelengths of light used). The $650 \mathrm{~nm}$ adapting light must affect both the middle- and long-wavelength receptors: thus under red adaptation the short-wavelength receptors will have a greater than normal relative contribution to the chromatic response. Since the short-wavelength cones contribute redness to the $r-g$ response. sensitivity changes alone can account for the empirical fact that the dark-adapted equilibrium blue wavelength at high intensities appears reddish-blue under moderate red chromatic adaptation (Cicerone et al.. 1975). This is indicated in Fig. 12 where, for each subject, the highest intensity equilibrium blue (for which the additive effect of the afterimage is minimized) is at a longer wavelength than the dark-adapted unique blue. While the sensitivity changes cause movement toward longer wavelengths, the greenness resulting from the afterimage requires compensating redness that. in the blue part of the spectrum, requires movement toward shorter wavelengths (Jameson and Hurvich. 1955). Thus as the intensity of the test field is decreased. the blue equilibrium lights should move toward shorter wavelengths. This is exactly what every subject's data show (Fig. 12). At low test intensities, the additive effect may neutralize or even exceed the wavelength shift induced by the sensitivity changes, in which case the red adapted equilibrium blue wavelength may be equal to or shorter than the dark- adapted setting. This seems to be the case for subjects TC and PS falthough measurement error prohibits a reliable inference with regard to the red adapted settings being below the comparable dark-adapted measurements).

The equilibrium blue experiments provide data that support the two-process theories by showing the predicted antagonism between the adaptation-induced additive and sensitivity change effects. These effects need not be antagonistic (the measurements of equilibrium yellow wavelengths are an example of when they are not). However. they frequently are antagonistic: in fact. in every experiment reported earlier in this study. the sensitivity changes and the additive effect tended to oppose each other. The possibility of antagonistic processes has the following implication for color perception: In general, the change in color appearance of a light (of watelength $i_{1}$ ). resulting from altering the adaptation state by adding to the adapting field light of watelength $i_{2}$. cannot be predicted knowing only the original color appearance of $i_{1}$ and the watelength $i_{2}$. For example. consider two yellow lights (that differ in brightness and or saturation) viewed under the same adaptation. There exist conditions where adding red light to the adapting field will cause one of the (previously) yellow lights to appear greenish and the other to appear reddish (see Figs +.6 and 8 ). Although the two-process theories can account for this result. this empirical finding is of course not dependent on any theoretical structure.

Ackmowledyements-This research was supported by National E: Institute grant EY07022-03 to The University of Micingan. and by the National Science Foundation through grant GB-366+2X to David H. Krantz and a graduate fellowship to the author. I am grateful to Dr Krantz for numerous discussions and many insightful suggestions concerning this research.

\section{REFEREYCES}

Brent R. P. (1973) Algorithms for Minimization without Dericatices. Prentice-Hall. Englewood Cliffs. New Jersey.

Cicerone C. M. Krantz D. H. and Larimer J. (1975) Opponent-process additivity-III. Effect of moderate chromatic adaptation. Vision Res. 15. 1125-1135.

Crawford B. H. (1947) Visual adaptation in relation to brief conditioning stimuli. Proc. R. Soc. 134B, 283-302.

De Valois R. L. and Walraven J. (1967) Monocular and binocular aftereffects of chromatic adaptation. Science 155. $463-465$.

Gestrin P. J. and Teller D. Y. (1969) Interocular hue shifts and pressure blindness. Vision Res. 9, 1267-1271.

Hering E. (1878) Zur Lehre rom Lichtsinne. Carl Gerold"s Sohn. Vienna.

Hurvich L. M. and Jameson D. (1958) Further development of a quantified opponent-colors theory. In Vistual Problems of Colour 11. pp. 691-723. HMSO. London. Jameson D. and Hurvich L. M. (1955) Some quantitative aspects of an opponent-colors theory. 1 . Chromatic responses and spectral saturation. J. opt. Soc. tm. 45. $546-552$.

Jameson D. and Hurvich L. M. (1959) Perceived color and its dependence on focal. surrounding and preceding stimulus variables. J. opt. Soc, Am. 49. 890-898.

Jameson D. and Hurvich L. M. (1961) Opponent chromatic induction: Experimental evaluation and theoretical account. J. opt. Soc. Am. 51, 46-53. 
Jameson D. and Hurvich L. M. (1964) Theory of brightness and color contrast in human vision. Vision Res. 4 . $135-154$.

Jameson D. and Hurvich L. M. (1972) Color adaptation: Sensitivity. contrast. and afterimages. In Hundbook of Sensory Physioloy! Vol. VII + (Edited by Jameson D. and Hurvich L. M. 1. pp. 568-581. Springer. Berlin.

Krantz D. H. (1975) Color measurement and color theory: II. Opponent-colors theory. J. math. Psichol. 12. $304-327$.

Kries J. von (1905) Die Gesichtsempfindungen. In Handbuch der Physiologie der. Menschen (Edited by Nagel W.). Springer. Berlin.

Larimer J.. Krantz D. H. and Cicerone C. M. (1974) Opponent-process additivity-1. Red green equilibria. $V$ ision Res. 14, 1127-1140.

MacAdam D. L. (1956) Chromatic adaptation. J. opt. Soc. 4m. 46, 500-513.

Moeller J. R. (1976) Measuring the redigreen quality of lights: A study relating the Jameson and Hurvich red green cancellation valence to direct magnitude estima- tion of greenness. Ph.D. dissertation. The University of Michigan.

Shevell S. K. (1977) The dual effect of backgrounds on color perception. Ph.D. dissertation. The University of Michigan.

Walraven J. (1973) Spatial characteristics of chromatic induction: the segregation of lateral effects from straylight artefacts. Vision Res. 13, 1739-1753.

Walraven J. (1976) Discounting the background-the miss. ing link in the explanation of chromatic induction. Vision Res. 16, 289-295.

Walters H. V. (1942) Some experiments on the trichromatic theory of vision. Proc. R. Soc. 131B, 27-50.

Westheimer G. (1966) The Maxwellian view. Vision Res. 6. 669-68?.

Whittle P. and Challands P. D. C. (1969) The effect of background luminance on the brightness of flashes. $V$ ision $R_{\mathrm{e}} \mathrm{s}$. 9, 1095-1109.

Wyszecki G. and Stiles W. S. (1967) Color Science: Concepts and Wethods, Quantitatice Data and Formulas. Wiley, New York. 